\title{
Development and application of the generic Plant growth Modeling Framework (PMF)
}

\author{
$\underline{\text { S. Multsch }}^{\text {a }}$, P. Kraft ${ }^{\text {a }}$ H.-G. Frede and L. Breuer ${ }^{\text {a }}$ \\ ${ }^{a}$ Institute for Landscape Ecology and Resources Management, Justus - Liebig - University Giessen \\ Email: Sebastian.Multsch@umwelt.uni-giessen.de
}

\begin{abstract}
The use of crop models as part of scientific research models or economic farm tools leads to a wide range of applications. On the one hand they need to be simple; on the other hand they should be complex enough to simulate a variety of growth mechanisms. The development of entirely new models for different questions requires a lot of coding and work such as changes in the model structure, the inclusion of alternative process descriptions or the implementation of additional functionality. Often, added model components do not really fit to the model philosophy of the originally developed base model.
\end{abstract}

We therefore developed a flexible (modular, generic and mixed procedural object oriented) and integrative (replaceable, expandable, independent and interactive) software tool for the setup of adapted crop models. The Plant growth Modeling Framework (PMF) is based on the Unified Modeling Language and implemented in Python, a high level object-oriented programming language. PMF provides the code flexibility to rapidly exchange and compare different process mechanisms. An interface facilitates a straightforward coupling with other models.

Two virtual case studies are presented to show the general functionality of PMF. In the first application PMF is coupled with a simple but widely used water balance model presented in the Food and Agricultural Organization FAO56 crop water guidelines. In contrast, the second case study focuses on the coupling of PMF with a complex Richard's equation based water balance model type created using the recently developed Catchment Modeling Framework (CMF). CMF follows the same philosophy as PMF, being flexible and integrative at the same time. In both case studies we show the tight connection between the water balance models and PMF and highlight the framework's ability to function as a model integrator while still being independent from the coupled models. This independency allows, for example, a further development of the different models (here the plant growth and the hydrological model components) by different research communities, which is often required in today's cross-disciplinary research consortia. Further, the modular and generic structure of PMF enables the use of process modules, which fit the level of complexity of the water balance models.

Besides aspects of architectural software development, PMF is equipped with an innovative interactive root growth mechanism. Root growth and branching is linked to soil depth dependent environmental conditions such as nitrogen supply or soil moisture. This reflects allows modelers to reflect the high interactivity of root (and plant) growth with soil environmental conditions. Thus, root elongation and root biomass allocation in PMF directly respond to changes of resource availability along the soil profile. This behavior is in close agreement with reality as plants grow where water, nutrients or other resources are available. We conclude that such a dynamic reaction on changes in resource availability improves the overall model credibility.

Keywords: Plant growth simulation, object oriented programming, model coupling, Python 


\section{INTRODUCTION}

Modeling of crop growth has a long tradition in agronomy. As early as the 1960s modeling concepts have evolved with a primary focus to improve the understanding of the underlying biological processes on the scale of single plants (Van Ittersum et al. 2003) and have been extensively reviewed by Bouman et al. (1996). Today, crop growth models are not only being used to predict the potential yield of crops or to study plant physiological processes. They are also part of many integrated models such as the water cycle in hydrological models, the energy exchange in soil-vegetation-atmosphere transfer schemes, agricultural decision support systems or the exchange of trace gases and carbon sequestration in biogeochemical models, to name only a few uses. The widespread utilization of crop models demands a flexible model, which can be integrated into model frameworks.

\subsection{Design of plant growth models}

The use of crop models as part of scientific research models or economic farm tools lead to a wide variety of applications, which need either sometimes requiring simple or solutions and other times more complex growth mechanisms. Models therefore need to be flexible with respect to processes and crop types that need to be represented. Three design characteristics describe such a flexible crop model (Reynolds and Acock 1997):

- Modularity: The model consists of replaceable modules, which have defined input and output values and represent different model mechanisms.

- Genericness: The modules should be independent from specific parameters and be applicable to several crop types.

- Mixed procedural-object oriented design: Modeling underlying growth process (e.g. development, potential growth, actual growth) is focused on the order of these processes. This approach is covered in a procedural programming approach. The division of the plant into its physical components focuses on the relationship between the plant organs. This second aspect is reflected in an objectoriented approach. The use of both approaches should lead to a well-structured software design.

One group of established crop growth models consists of the 'School of de Wit' models. This group includes the crop models SUCROS and WOFOST which have a modular, procedural and generic design. A second group is the CERES-family such as the wheat simulator SIRIUS or the legume production model CROPGRO. These models have a modular, procedural and non-but no generic design, and are, for that reason, related to a specific crop. Whereas these crop models have been mainly developed for the prediction of plot to field scale crop growth, additional models have primarily been designed for larger scale application, such as EPIC or CREAMS. By contrast a third group refer to new crop model types, which have a modular and generic structure and combine procedural and object-oriented programming approaches such as GePSI, APSIM-GCROP, SPASS, or STICS. They are characterized by exchangeable environmental and process modules and are mainly based on components already established in the School of de Wit and CERES crop models.

Many crop growth models are used in landscape or catchment scale tools, for example in the spatial Decision Support Systems DSSAT4.0, or in the hydro-biogeochemical models SWAP and DAISY. The widely used water quality model SWAT includes a simplified version of EPIC. A typical feature of all these models is that the incorporated crop models act as subordinate modules with a fixed code connection. A more flexible way of integration can be realized within modular model frameworks (Wang and Engel 2000). This approach enables the development and variation of each model without changing the other models.

\subsection{Design of model frameworks}

The idea of model frameworks is that they integrate models from different scientific disciplines such as hydrology, soil science, meteorology, plant physiology, or others in an independent way. For this reason integrative model frameworks must conform to several design characteristics:

- Replaceability comprises the easy adaption of model components due to new scientific knowledge (Timlin and Pachepsky 1997).

- Expandability allows the addition of model components from other scientific disciplines, while each model can be developed by the respective experts (Timlin and Pachepsky 1997).

- Independency is necessary to connect models on an abstract level, e.g. by interfaces. This enables a fast and easy data transfer. A variation of a single model leads to a variation of the interface without the needs of changing other models (Timlin and Pachepsky 1997). 
- Interactivity (or connectability) states that coupled models should be highly interactive, i.e. each model should be open for influences from other independent models (Kraft et al., 2010).

In summary the design characteristics listed above represent the requirements for the development of a flexible (modular, generic, mixed object-oriented and procedural) and integrative (replaceable, expandable, independent, connectable) crop model. On this basis we developed the Plant growth Modeling Framework (PMF). In the following we present its structure, functionalities and show results of two case studies.

\section{DESIGN OF THE PLANT GROWTH MODELING FRAMEWORK (PMF)}

PMF follows the design characteristics of flexibility and integrativeness. It considers the advantages of modularity and enables the use of generic mathematical solutions with different levels of complexity. To achieve this, PMF creates a data transfer scheme with the "has a relationship" programming approach, which enables data exchange of state variables and boundaries, without implementing the underlying biophysical process. The mixed procedural-object oriented design facilitates replacement of (crop) model processes (replaceability) and provides an expandable model structure that can be easily refined due to new knowledge, improved process understanding or additional modeling objectives (expandability). PMF is able to exchange data with models that have a different level of complexity and allows retrieving data for superordinate systems (independency). Interfaces facilitate the data connection to other models, which leads to an independent and connectable structure (interactivity). In consequence of the tight connection to other models, PMF is highly interactive within short time steps, e.g. plant stress adaption due to drought and nitrogen stress in the early growing season.

The development of PMF is based on the Unified Modeling Language (UML), which allows the set up of an object oriented modeling structure. The benefit of UML for creating a modular or component-based approach in environmental crop modeling is discussed by Papajorgji et al. (2004). They explicitly pinpoint the advantage of an improved exchange and transfer of knowledge when several modeling teams from various disciplines are involved in setting up an integrated model. PMF is implemented with the high level scripting language Python. This programming language has a widespread utilization in scientific modelling and enables the development of an object-oriented structure. PMF is coupled by predefined interfaces with other models. The potential use of scripting languages as "glue" between different models has been proposed from Ousterhout (1998) and Python is used in accompanying model frameworks for hydrology (Kraft et al., 2011).

\subsection{Overall model configuration}

PMF consists of four core-modules (Figure 1), the Plant Model, the Process Library, the Crop Database and the Plant Building Set. Each module contains different classes. The Plant Model represents the abstract physiological connection of the structural organs and the related growth processes. The Process Library implements biophysical processes as a collection of independent classes, which define methods of substrate exchange inside the plant, the uptake of water and nutrients or the plant development. The Crop Database contains all relevant crop specific parameters that are required to initialize and run the model. The Plant Building Set links the Process Library and the Crop Database to the Plant Model.

\subsection{Setup of a model run}

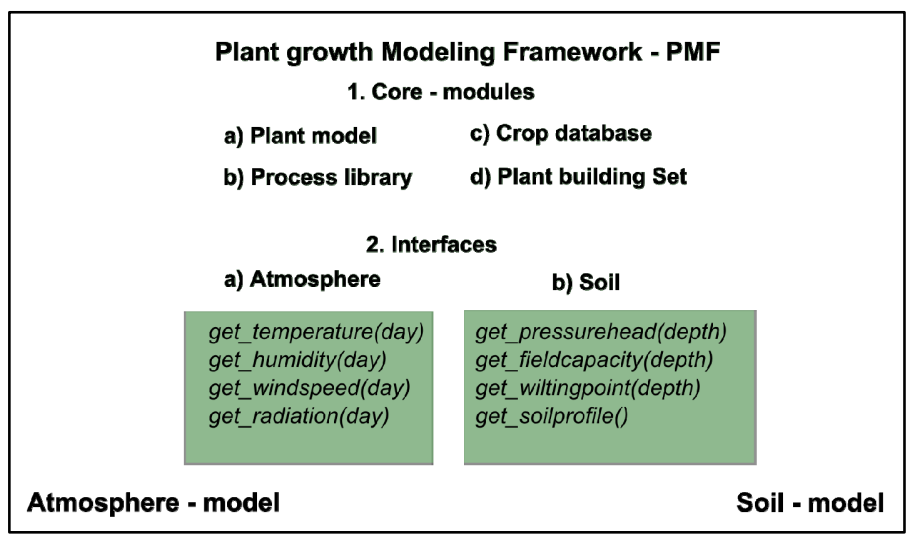

Figure 1. Components of PMF. The core-modules include the Plant model (1a), which is described by an abstract growth structure. This structure is linked to mathematical solutions of processes contained in the Process library (1b). The Crop database (1c) holds relevant plant specific parameters. The three components are connected through the Plant building set

The Setup script in Figure 2a defines the surrounding requirements for a PMF simulation and calls the Runtime loop (Figure 2b). In the first four run time steps the soil and the atmosphere interfaces from PMF are implemented. The interfaces can be represented as static databases or dynamic models. The setup script in the example implements a water balance model as the soil interface and loads meteorological data from a static 
database as the atmosphere interface. The next two steps specify the simulation setup. If PMF runs without a management model, the sowing and harvest dates must be implemented from the user. In the next step the simulation period is defined and the runtime loop is called. The Runtime loop in Figure $2 \mathrm{~b}$ controls the run of processes during the simulation period. This includes the control of conditions, which initialize growth and management processes. The sowing process includes the creation of a plant object with the Plant building set. In the next step the Plant object is called and the calculation procedure proceeds. PMF, the soil and the atmosphere model calculate the state variables in each time step. The fluxes and substrates between the models are handled by the interfaces.

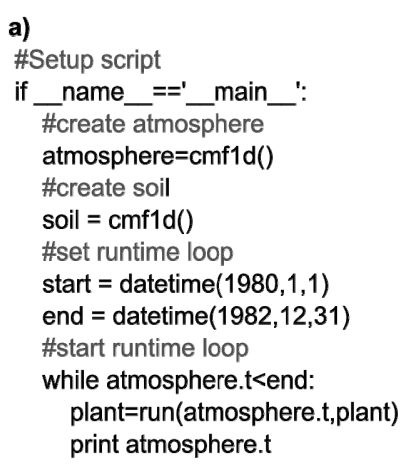

Figure 2. Code snippets from the Setup script (a) and the Runtime loop (b) of a PMF setup with CMF.

\section{MODEL SETUP FOR VIRTUAL CASE STUDIES}

\subsection{General set up of PMF}

The general functionality of PMF and its high interactivity with other models will be shown in two case studies, which have been set up in a virtual experiment fashion. Such experiments allow testing models in a numerical way, by driving the model with 'collective field intelligence' as outlined by Weiler and McDonnell (2004). In the following case studies, PMF has been coupled with two kinds of water balance models, using the same Plant model setup but different water stress functions. The aim of the first case study is to present the interactions between soil water supply and plant growth in relation to the growing season. The second case study shows the dynamic and interactive root growth along a drought event in the early growing season with a high water loss in the upper soil layers.

In both virtual experiments meteorological data from the weather station Giessen, Germany, have been used. The soil physical properties are related to sand, clay and silt content of $20 \%, 20 \%$, and $60 \%$ respectively. They represent a typical soil Loess substrate from Germany. Summer wheat has been selected as the dominant crop for PMF, using the following parameter set for the general processes:

- Development stages and related thermal time values are taken from Miller et al. (2001). The development is divided into emergence (160 growing degree days, GDD), leaf development (208 GDD), tillering (421 GDD), stem elongation (659 GDD), anthesis (901 GDD), seed fill (1174 GDD), drought stage (1556 GDD) and maturity (1665 GDD). $\mathrm{T}_{\text {base }}$ is set to zero.

- Biomass accumulation is calculated by the light-use efficiency (LUE) concept with a LUE of $3.0 \mathrm{~g} \mathrm{~m}^{-2} \mathrm{MJ}^{-1}$ and an extinction coefficient of 0.4 as described by Acevedo et al. (2002).

- The values for the calculation of the crop specific evapotranspiration are taken from Allen et al. (1998): the end of the initial growth stage, crop development stage, mid season and late season must be known. The end of these seasons is defined in relation to the seven development stages given above. Initial growth stage refers to the end of emergence, crop development stage to the end of tillering, mid season to the end of anthesis and the late season to the beginning of physiological maturity. The related basal crop coefficients $\left(\mathrm{K}_{\mathrm{cb}}\right)$ are $\mathrm{K}_{\mathrm{cb}}$ ini $0.15, \mathrm{~K}_{\mathrm{cb}}$ mid 1.1 and $\mathrm{K}_{\mathrm{cb}}$ end 0.15 .

- The root properties refer to a maximum rooting depth of $150 \mathrm{~cm}$ and the vertical growth rate of $1.5 \mathrm{~cm}$ day $^{-1}$.

- Relative field cover is calculated from the actual leaf area index (LAI) in relation to a full field cover assumed at a LAI of $3.0 \mathrm{~m}^{2} \mathrm{~m}^{-2}$ (De Vries et al. 1989).

- A specific leaf weight of $40 \mathrm{~g} \mathrm{~m}^{-2}$ is assumed for the calculation of the LAI based on dry leaf biomass (De Vries et al. 1989).

- The maximum nitrogen content decreases from $4.3 \%$ at emergence to $1.6 \%$ at maturity (Jamieson and Semenov 2000). 


\subsection{Specific set up for individual soil water models}

The soil water flux processes vary with the implemented soil water model. In the first setup, PMF is coupled to the soil water balance model of FAO56, which is based on numerical solutions described by Allen et al. (1998). The soil physical processes are described through the fraction of sand, clay and silt. From these values, the water content at saturation, field capacity and wilting point is determined. The plant root is equally distributed over the rooting depth. In the second setup PMF is coupled to a one dimensional multilayer soil setup of the Catchment Modeling Framework (Kraft et al. 2011). In this more complex model soil water model, the soil is divided into 40 soil layers with a thickness of $0.05 \mathrm{~m}$ each. Soil hydrological conditions are represented by the Brooks-Corey expression, based on field capacity, porosity and saturated hydraulic conductivity. These values are derived from the above given sand, clay and silt content in each soil layer, assuming no change with depth. The water fluxes between the soil layers are described by the Richards' equation, both under unsaturated as well as saturated conditions. Water uptake is restricted in relation to the pressure head in each soil layer, whereby a stress response function describes plant water stress due to dry and wet conditions. This approach has been introduced by Feddes et al. (1978).

\section{COMPARISON OF A SIMPLE VERSUS A MORE COMPLEX SOIL WATER MODEL}

PMF has been coupled with the simple FAO56 water balance model (case study I) and the more complex CMF (case study II). The simulation includes the time period from 1980 to 2000 . Results of both simulations are given in Table 1 .

Table 1. Results of coupling PMF with the two water balance models. $\mathrm{W}_{\text {pot }}$ and $\mathrm{W}_{\text {act }}=$ potential and actual biomass; early stress = time period from emergence to anthesis; late stress = time period from anthesis to physiology maturity; LAI = leaf area index; $\mathrm{Z}_{\mathrm{r}}=$ rooting depths

\begin{tabular}{|c|c|c|c|c|c|c|c|}
\hline & & $\begin{array}{l}\mathbf{W}_{\text {pot }} \\
{[g}\end{array}$ & $\mathbf{W}_{\text {act }}$ & Early stress & Late stress & $\underset{\left[\mathrm{m}^{2} \mathrm{~m}^{-2}\right]}{\mathbf{L A I}}$ & $\begin{array}{c}\mathbf{Z}_{\mathbf{r}} \\
{[\mathrm{cm}]}\end{array}$ \\
\hline \multirow[t]{3}{*}{$\begin{array}{l}\text { Case study I } \\
\text { (FAO56) }\end{array}$} & Average & 1840 & 1705 & 3 & 12 & 4.2 & 124 \\
\hline & Min & 1217 & 873 & 0 & 0 & 2.3 & 110 \\
\hline & $\operatorname{Max}$ & 2330 & 2211 & 12 & 37 & 6.1 & 150 \\
\hline \multirow[t]{3}{*}{$\begin{array}{l}\text { Case study II } \\
\text { (CMF) }\end{array}$} & Average & 1823 & 1319 & 6 & 32 & 4.1 & 94 \\
\hline & Min & 1217 & 805 & 0 & 2 & 2.3 & 71 \\
\hline & $\operatorname{Max}$ & 2236 & 2160 & 5 & 44 & 6.1 & 124 \\
\hline
\end{tabular}

In general, the setup with CMF predicts slightly lower values for biomass allocation in comparison to the FAO56 model, whereby the lowest predicted value for $\mathrm{W}_{\text {pot }}$ is $1217 \mathrm{~g} \mathrm{~m}^{-2} \mathrm{a}^{-1}$ for both set ups. The highest value for $W_{p o t}$ is $2230 \mathrm{~g} \mathrm{~m}^{-2} \mathrm{a}^{-1}$ as simulated by FAO56. In both setups similar values are predicted for $L A I$ with an average value of around $4.1 \mathrm{~m}^{2} \mathrm{~m}^{-2}$. The rooting depth $Z_{r}$ differs between the two models, whereby the values of CMF are about $0.25-0.40 \mathrm{~m}$ lower in comparison to the FAO56 water balance model. These differences can be explained by the higher spatial resolution simulation of soil layer specific root growth and its interaction to environmental conditions in CMF (40 soil layers) as compared to FAO56 (1 bulk soil layer). Differences in root elongation can lead to the development of a different number of stress days, however, other processes such as variation in actual evaporation between the two set ups can also lead to differences in stress.

Water stress limits biomass accumulation, restricts root growth and leaf development. In PMF, the $1^{\text {st }}$ period of plant growth dominates leaf development and root growth and the $2^{\text {nd }}$ period gives more weight to biomass accumulation. Table 1 shows the sum of days, where the decrease in biomass accumulation due to water stress exceeds $10 \%$. In general, water limiting conditions occur more often in the later period, with maximum values of 37 and 44 days for FAO56 and CMF, respectively. One explanation is the reduced rooting depth and the restriction of plant water uptake from deeper soil layers in the CMF set up. 


\section{IMPACT OF WATER STRESS ON ROOT GROWTH}

To further investigate the effect of environmental conditions on root growth, we additionally implemented soil water stress in case study II. For this, we assume that the upper three soil layers $(0-15 \mathrm{~cm})$ dry out in the early growing season by high evaporation. The effect on the development of daily root biomass, annual root biomass allocation and water uptake for 0 to $1.0 \mathrm{~m}$ soil depth is shown in Figure 4 . Figure $4 \mathrm{a}$ and $4 \mathrm{~b}$ depict the results without the influences from drought stress, whereas a drought stress effect is included in results of Figure $4 \mathrm{c}$ and $4 \mathrm{~d}$. The highest daily root biomass increase can be observed in the upper soil layers with values of around 9 to $12 \mathrm{~g}_{\text {layer }}{ }^{-1} \mathrm{day}^{-1}$. The annual root water uptake is around 30 to $60 \mathrm{~mm} \mathrm{~cm}^{-1}$ in the upper soil layer and decreases with depth. The annual (total) root growth shows a similar behaviour, with lowest values at a depth of around $1 \mathrm{~m}$, where the plant reaches its maximum rooting depth. The lower two graphs of Figure 4 show the results of simulated root growth and water uptake with a drought stress occurring in the early growing season. Here, the highest daily root biomass increase is located in the fourth and the fourth soil layer with values of $3.5 \mathrm{~g}$ to $6 \mathrm{~g}$ layer $^{-1} \mathrm{day}^{-1}$.

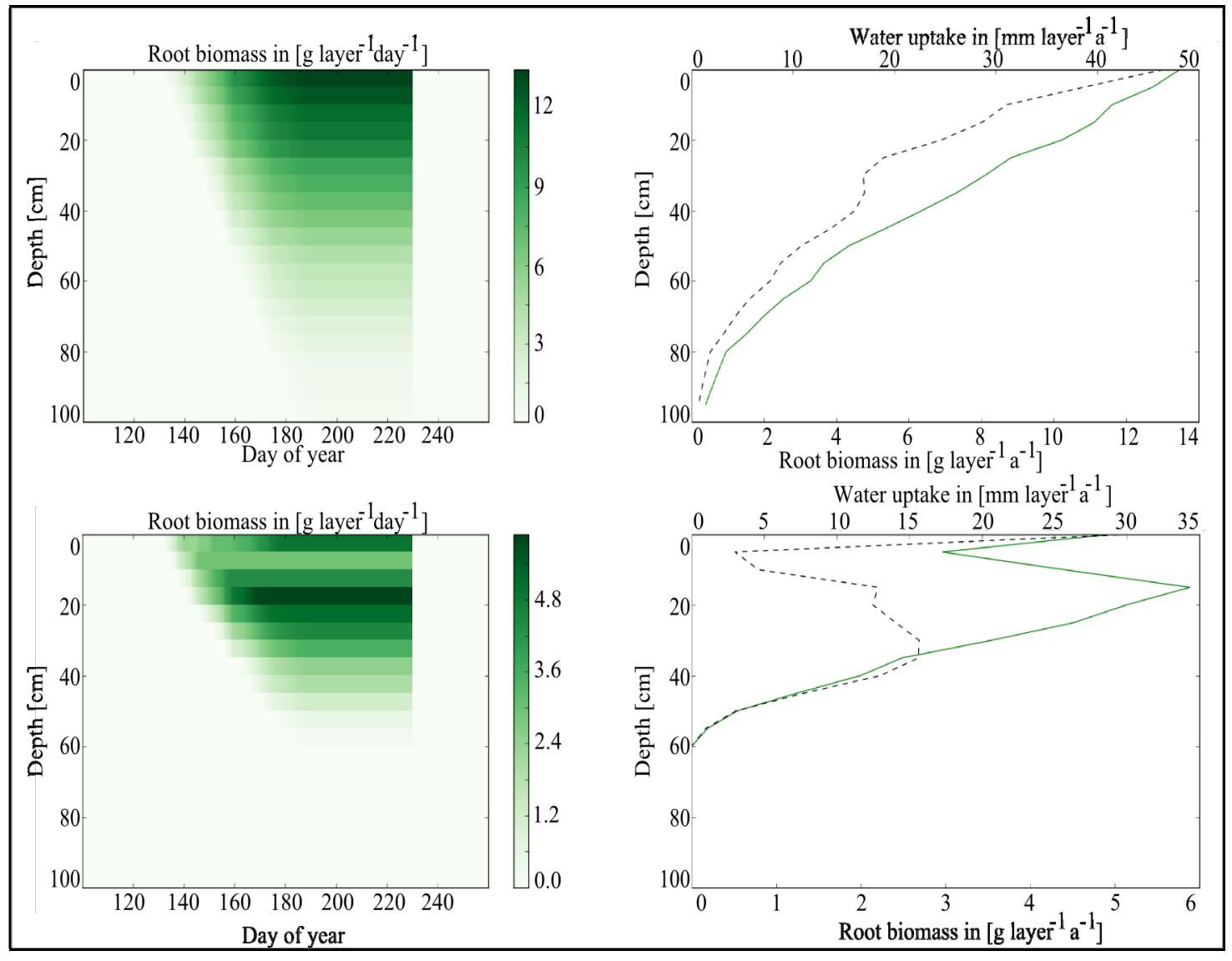

Figure 4. Simulations without ( $a$ and $b$ ) and with (c and d) a drought stress event in the early growing season. Graphs a and c show the daily root biomass distribution in relation to depth and day of year. Graphs b and d depict the cumulative root biomass allocation (solid line) and the water uptake (dashed line) per layer over the growing season.

\section{CONCLUSIONS}

With respect to the design characteristics of flexible crop models (modularity, genericness, mixed procedural object oriented approach) we set up the Plant growth Model Framework (PMF). The model was coupled to independent soil water models in two case studies. We showed that PMF is able to work in a tight interactive model connection while still being independent from the coupled models. The modular and generic structure of PMF enables the use of process modules, which fit to the level of complexity of the water balance models. 
Apart from the general functionalities of PMF we were able to show that a high interactivity of PMF with environmental boundary conditions (soil moisture status) can be simulated within the proposed model framework. Root growth and root biomass allocation in PMF directly respond to changes of resource availability along the soil profile. This is in close agreement with the observation that plants grow where water, nutrients or other resources such as light are available. We conclude that such a dynamic reaction on changes in resources availability improves the overall model credibility.

The two case studies presented here are based on a virtual experimental set up. For a further proof-ofconcept, PMF needs to be tested with field data under realistic boundary conditions, preferably in a model inter-comparison approach that has been conducted for several other models (Eitzinger et al. 2004; Wolf et al. 1996). This would also allow investigating the structural differences of the various crop growth models that exist in the field of agronomy. To better compare the capability of crop growth models we also advocate setting up a publicly available benchmark data set.

\section{REFERENCES}

Acevedo, E., Silva, P. and Silva, H. (2002). Wheat growth and physiology. Curtis, B. C.; Rajara, S.; Macpherson, H. G. (eds.). Bred Wheat - improvement and production. Rome: FAO, 39-70.

Allen, R. G., Pereira, L. S., Raes, D. and Smith, M. (1998). Crop evapotranspiration-Guidelines for computing crop water requirements. FAO Irrigation and Drainage Paper 56, Rome, Italy.

Bouman, B. A. M., van Keulen, H., van Laar, H. H. and Rabbinge, R. (1996). The School of de Wit crop growth simulation models: a pedigree and historical overview. Agricultural Systems, 52(2-3), 171-198.

Feddes, R. A., Kowalik, P. J. and Zaradny, H. (1978). Simulation of field water use and crop yield. Wageningen: Centre for Agricultural Publishing and Documentation.

Van Ittersum, M. K., Leffelaar, P. A., Van Keulen, H., Kropff, M. J., Bastiaans, L. and Goudriaan, J. (2003). On approaches and applications of the Wageningen crop models. European Journal of Agronomy, 18(34), 201-234.

Kraft, P., Multsch, S., Vaché, K. B., Frede, H. G. and Breuer, L. (2010). Using Python as a coupling platform for integrated catchment models. Advances in Geosciences, 27, 51-56.

Kraft, P., Vaché, K. B., Frede, H. G. and Breuer, L. (2011). A hydrological programming language extension for integrated catchment models. Environmental Modelling \& Software, 26, 828-830.

Miller, P., Lanier, W. and Brandt, S. (2001). Using growing degree days to predict plant stages. $\mathrm{Ag}$ /Extension Communications Coordinator, Communications Services, Montana State UniversityBozeman, (7).

Ousterhout, J. K. (1998). Scripting: Higher-level programming for the 21st century. IEEE Computer, 31(3), 23-30.

Papajorgji, P., Beck, H. W. and Braga, J. L. (2004). An architecture for developing service-oriented and component-based environmental models. Ecological Modelling, 179(1), 61-76.

Reynolds, J. F. and Acock, B. (1997). Modularity and genericness in plant and ecosystem models. Ecologica Modelling, 94(1), 7-16.

Timlin, D. J. and Pachepsky, Y. A. (1997). A modular soil and root process simulator. Ecological Modelling, 94(1), 67-80.

De Vries, P., Balema, A., Jansen, D. M. and Ten Berge, H. F. M. (1989). Simulation of ecophysiological process of growth in several annual crops. Center for agriculture publishing and documentation (Pudoc), Wageningen.

Wang, E. and Engel, T. (2000). SPASS: a generic process-oriented crop model with versatile windows interfaces. Environmental Modelling and Software, 15(2), 179-188.

Weiler, M. and McDonnell, J. (2004). Virtual experiments: a new approach for improving process conceptualization in hillslope hydrology. Journal of Hydrology, 285(1-4), 3-18. 